\title{
Resveratrol alleviates hypoxia/reoxygenation injury-induced mitochondrial oxidative stress in cardiomyocytes
}

\author{
TAO LI ${ }^{1 *}$, LINLIN CHEN $^{1,2^{*}}$, YIYAN YU ${ }^{1}$, BINBIN YANG $^{1}$, PENGYUN LI $^{1}$ and XIAO-QIU TAN ${ }^{1}$ \\ ${ }^{1}$ Key Laboratory of Medical Electrophysiology of Ministry of Education, \\ Collaborative Innovation Center for Prevention and Treatment of Cardiovascular Disease, \\ Institute of Cardiovascular Research, Southwest Medical University; ${ }^{2}$ Department of Cardiovascular Medicine, \\ Sichuan Luzhou TCM Hospital, Luzhou, Sichuan 646000, P.R. China
}

Received July 3, 2018; Accepted January 29, 2019

DOI: $10.3892 / \mathrm{mmr} .2019 .9943$

\begin{abstract}
Resveratrol (RES) is a naturally occurring antioxidant compound found in red wine. Although it has been demonstrated to have a cardioprotective effect, the mechanism underlying this effect remains to be fully elucidated. The aim of the present study was to determine whether RES exerts a protective effect against mitochondrial oxidative stress and apoptosis in neonatal rat cardiomyocytes (NRCMs) induced by hypoxia/reoxygenation (H/R) injury. Primary cultured NRCMs were used as a model system and were divided into four experimental groups: Control, H/R, H/R + DMSO (H/R with $0.2 \% \mathrm{DMSO})$ and $\mathrm{H} / \mathrm{R}+\mathrm{RES}(\mathrm{H} / \mathrm{R}$ with $100 \mu \mathrm{M} \mathrm{RES})$ groups. Mitochondrial oxidative stress was determined by measuring the alteration in the mitochondrial membrane potential $(\Delta \Psi \mathrm{m})$ of NRCMs, the release of lactate dehydrogenase (LDH) and the ratio of $\mathrm{B}$-cell lymphoma 2 (Bcl-2)/Bcl-2-associated $\mathrm{X}$ protein (Bax) from NRCMs. Cell apoptosis was assessed by measuring cell apoptotic rates and the activity of caspase 3 . In the H/R+RES group, RES significantly alleviated structural impairment, including disordered $\alpha$-actin and $\mathrm{F}$-actin, in the NRCMs induced by H/R injury. RES attenuated H/R injury-induced mitochondria oxidative stress. RES also attenuated H/R injury-induced cell apoptosis; it decreased the NRCM apoptotic rate from $84.25 \pm 7.41 \%$ (H/R) to $46.39 \pm 5.43 \%$ $(\mathrm{H} / \mathrm{R}+\mathrm{RES})(\mathrm{P}<0.05, \mathrm{n}=4)$, rescued the decrease in the Bcl2/Bax ratio induced by $\mathrm{H} / \mathrm{R}$ from $0.53 \pm 0.08$-fold $(\mathrm{H} / \mathrm{R})$ to $0.86 \pm 0.06$-fold $(\mathrm{H} / \mathrm{R}+\mathrm{RES})(\mathrm{P}<0.05, \mathrm{n}=5)$ and alleviated
\end{abstract}

Correspondence to: Dr Xiao-Qiu Tan, Key Laboratory of Medical Electrophysiology of Ministry of Education, Collaborative Innovation Center for Prevention and Treatment of Cardiovascular Disease, Institute of Cardiovascular Research, Southwest Medical University, 319 Zhongshan Road, Luzhou 646000, P.R. China

E-mail: tanxiaoqiu@swmu.edu.cn

*Contributed equally

Key words: resveratrol, cardiomyocyte, hypoxia/reoxygenation, mitochondria oxidative stress, sirtuin 1 the increased activity of caspase 3 induced by $\mathrm{H} / \mathrm{R}$ from $1.32 \pm 0.06$-fold to $1.02 \pm 0.04$-fold $(\mathrm{P}<0.05, \mathrm{n}=5)$. Furthermore, RES significantly attenuated the increment of LDH release induced by $\mathrm{H} / \mathrm{R}$ injury in NRCMs from $1.41 \pm 0.03$-fold (H/R) to $1.02 \pm 0.06$-fold $(\mathrm{H} / \mathrm{R}+\mathrm{RES})(\mathrm{P}<0.01, \mathrm{n}=4)$ and alleviated the depolarization of $\Delta \Psi \mathrm{m}$ induced by $\mathrm{H} / \mathrm{R}$, shifting the ratio of JC-1 monomer from $62.39 \pm 1.82 \%(\mathrm{H} / \mathrm{R})$ to $35.31 \pm 8.63 \%$ $(\mathrm{H} / \mathrm{R}+\mathrm{RES})(\mathrm{P}<0.05, \mathrm{n}=4)$. RES alleviated the decrease in sirtuin 1 induced by $H / R$ injury from $0.61 \pm 0.06$-fold $(H / R)$ to $1.01 \pm 0.05$-fold $(\mathrm{H} / \mathrm{R}+\mathrm{RES})(\mathrm{P}<0.05, \mathrm{n}=5)$. In conclusion, the present study is the first, to the best of our knowledge, to demonstrate that RES provides cardioprotection against H/R injury through decreasing mitochondria-mediated oxidative stress injury and structural impairment in NRCMs. These results provide scientific evidence for the clinical application of RES in the treatment of cardiac conditions.

\section{Introduction}

Myocardial ischemia/reperfusion (I/R) injury is a major pathophysiological condition associated with cardiopulmonary bypass surgery and various cardiovascular diseases (CVDs), including coronary heart disease and myocardial infarction, both of which have high mortality and morbidity rates $(1,2)$. Mitochondria are the organelles providing the major source of reactive oxygen species (ROS) production and are rich in cardiomyocytes $(3,4)$. Mitochondrial oxidative stress serves a central role in myocardial I/R injury. Hypoxia induces superoxide generation via the electron transport chain. High concentrations of ROS disrupt cell functions, induce apoptosis and cause tissue damage via inducing oxidative stress. Therefore, mitochondria-mediated oxidative stress is the key step in I/R-induced cardiomyocytes injury $(5,6)$. It is also a main target for designing treatment strategies for I/R-induced cardiomyocyte injury.

Resveratrol (RES), a natural compound found primarily in red wine, is a naturally occurring antioxidant. The potential beneficial effects of RES were first observed in major CVDs, including myocardial ischemia, atherosclerosis, hypertension, stroke, aging and heart failure (7). Previous studies have revealed that the cadioprotective effects of RES include anti-oxidative stress, antiplatelet activity, preventing 
endothelial cell damage and inflammation, and increasing the expression of nitric oxide synthase. The biological effects of RES, including chemoprevention, immunomodulatory, antiproliferative and antioxidant effects, have been reported previously (8-11). Recently, it was reported that RES exerts its cardioprotective effect through the AMP-activated protein kinase and phosphoinositide-3-kinase/Akt/Forkhead box O3a signaling pathways $(12,13)$. Additionally, it was reported that pre-treatment with RES may prevent ischemic cerebral damage in rats by activating sirtuin 1 (Sirt1), one of the ubiquitous histone and protein deacetylases (14). RES-mediated autophagy is also involved in cardioprotection (15). However, the mechanisms involved in the cardioprotective effect of RES remain to be fully elucidated. The mitochondria-mediated oxidative stress pathway is one of the major causes of $\mathrm{I} / \mathrm{R}$ or hypoxia/reoxygenation $(\mathrm{H} / \mathrm{R})(4,16)$. ROS are generated in the $\mathrm{I} / \mathrm{R}$ or $\mathrm{H} / \mathrm{R}$ myocardium, particularly in mitochondria. I/R injury has been associated with significant increases in ROS, the release of lactate dehydrogenase (LDH) and depolarization of the mitochondrial transmembrane potential $(\Delta \Psi \mathrm{m})$, which are all important indices for reflecting the status of mitochondrial oxidative stress. Enhanced mitochondrial oxidative stress induces cell injury and apoptosis $(17,18)$. Previous findings have also shown that RES has anti-oxidant properties (19). Hypoxia is an important pathophysiological progress and induces cell injury through crosstalk with mitochondria and oxidative stress pathways (20). Sirt1 is also involved in hypoxia-induced pulmonary artery smooth muscle cell injury (21). To gain further insight into the mechanisms underlying the RES-mediated protective effect against I/R injury, an $\mathrm{H} / \mathrm{R}$ model of cultured NRCMs was established in the present study. Using this model, the cardioprotective effect of RES and the underlying mechanism were examined. The results showed that RES alleviated H/R injury through inhibiting the mitochondria-mediated oxidative stress pathway. This provides a robust scientific basis for the clinical application of RES in the treatment of cardiac conditions.

\section{Materials and methods}

Drugs and reagents. RES (molecular formula: $\mathrm{C}_{14} \mathrm{H}_{12} \mathrm{O}_{3}$; CAS no. 501-36) was purchased from Neptunus Company (Shenzhen, China). A MitoProbe ${ }^{\mathrm{TM}}$ JC-1 Assay kit (cat. no. M34152) for measuring the $\Delta \Psi \mathrm{m}$ was obtained from Thermo Fisher Scientific, Inc. (Waltham, MA, USA). Dimethyl sulfoxide (DMSO) was purchased from Sigma; Merck KGaA (Darmstadt, Germany). The BCA protein assay (cat. no. 23225) was purchased from Thermo Fisher Scientific, Inc. All other reagents and chemicals were obtained commercially and were of analytical grade.

Cell culture and the treatment. The experimental protocols were performed in accordance with the National Institutes of Health Guide for the Care and Use of Laboratory Animals (National Institutes of Health, Bethesda, MD, USA) and with the approval of the Animal Care and Use Committee of the Southwest Medical University (Sichuan, China).

Neonatal rats (age, 2-3 days; weight, 5-8 g; n=150) were purchased from The Animal Center of Southwest Medical University (Luzhou, China). Neonatal rats were maintained at
$22 \pm 3^{\circ} \mathrm{C}$ under a 12 -h light/dark cycle. All rats had free access to water and food. Primary cultured neonatal rat cardiomyocytes (NRCMs) were cultured as previously described (22). In brief, neonatal rat ventricles were digested with collagenase II and cardiomyocytes were purified through adherence to culture plastic for different durations. Subsequently, $0.1 \mathrm{mM}$ 5-BrdU was added to DMEM to inhibit the cardiac fibroblasts. The NRCMs were cultured in DMEM (cat. no. SH30021; HyClone; GE Healthcare Life Sciences, Logan, UT, USA) containing 10\% FBS (cat. no. 10270; Gibco; Thermo Fisher Scientific, Inc.) and $1 \%$ penicillin/streptomycin (cat. no. C0222; Beyotime Institute of Biotechnology, Haimen, China) at $37^{\circ} \mathrm{C}$ in a $5 \%$ $\mathrm{CO}_{2}$ incubator. The NRCMs were randomly divided into four groups: i) Control group (normal DMEM); ii) H/R group: Cells were subjected to a completely enclosed environment containing a Bio-Bag (Thermo Fisher Scientific, Inc.) for $8 \mathrm{~h}$ in hypoxic medium and then subjected to reoxygenation under normoxic conditions for another $8 \mathrm{~h}$, following which the hypoxic medium was replaced with fresh medium (containing $10 \% \mathrm{FBS}$ ) upon reoxygenation; iii) $\mathrm{DMSO}+\mathrm{H} / \mathrm{R}$ group: $0.2 \%$ DMSO was added to the medium during $H / R$; iv) $\mathrm{RES}+\mathrm{H} / \mathrm{R}$ group: RES (final concentration of $100 \mu \mathrm{M}$ ) was added to the medium during $\mathrm{H} / \mathrm{R}$.

Measurement of $\mathrm{LDH}$ release. $\mathrm{LDH}$ release was detected using an LDH assay kit (cat. no. A020, Nanjing Jiancheng Bioengineering Institute, Nanjing, China) according to the manufacturer's protocol. In brief, the NRCMs were subjected to centrifugation at $300 \mathrm{xg}$ for $10 \mathrm{~min}$. Following this, $60 \mu \mathrm{l}$ of supernatant was added to $30 \mu \mathrm{l}$ of $\mathrm{LDH}$ substrate solution and incubated for $30 \mathrm{~min}$ at $37^{\circ} \mathrm{C}$. The $\mathrm{LDH}$ levels were detected with a microplate reader (Tecan Group, Inc., Mannedorf, Switzerland) at $440 \mathrm{~nm}$.

Measurement of $\Delta \Psi m$. The $\Delta \Psi \mathrm{m}$ was measured by flow cytometry using the MitoProbe ${ }^{\mathrm{TM}} \mathrm{JC}-1$ assay kit. The approximate excitation peak of JC-1 is $488 \mathrm{~nm}$. The approximate emission peaks of monomeric and J-aggregate forms are 529 and $590 \mathrm{~nm}$, respectively. The ratio of monomeric JC-1 represents the level of $\Delta \Psi \mathrm{m}$. An increased ratio represents the depolarization of $\Delta \Psi \mathrm{m}$, whereas a decreased ratio indicates the hyperpolarization of $\Delta \Psi \mathrm{m}$.

Immunofluorescence microscopy. Primary cultured NRCMs were plated on the coverslips at $60-70 \%$ confluency and fixed in cold PBS with paraformaldehyde for $15 \mathrm{~min}$, permeabilized with $0.1 \%$ triton-X 100 for 15 min and blocked with 5\% bovine serum albumin (cat. no. a600332; BBI Life Sciences Corporation, Shanghai, China) for $1 \mathrm{~h}$ at room temperature. The NRCMs were then incubated with primary mouse anti- $\alpha$-actinin 2 antibody (1:100; cat. no. BM4907; Wuhan Boster Biological Technology, Ltd., Wuhan, China) overnight at $4^{\circ} \mathrm{C}$ and then incubated with the DyLight ${ }^{\circledR} 488$-conjugated donkey anti-mouse secondary antibody (1:200; cat. no. ab96875; Abcam, Cambridge, UK) for $1 \mathrm{~h}$ at room temperature. In addition, for the staining of F-actin, the NRCMs were treated with $100 \mathrm{nM}$ rhodamine phalloidin (cat. no. PHDR1; Cytoskeleton, Inc., Denver, CO, USA) for $30 \mathrm{~min}$ at room temperature. The nuclei were stained with DAPI. The cells were mounted and images were captured using an Olympus fluorescence 

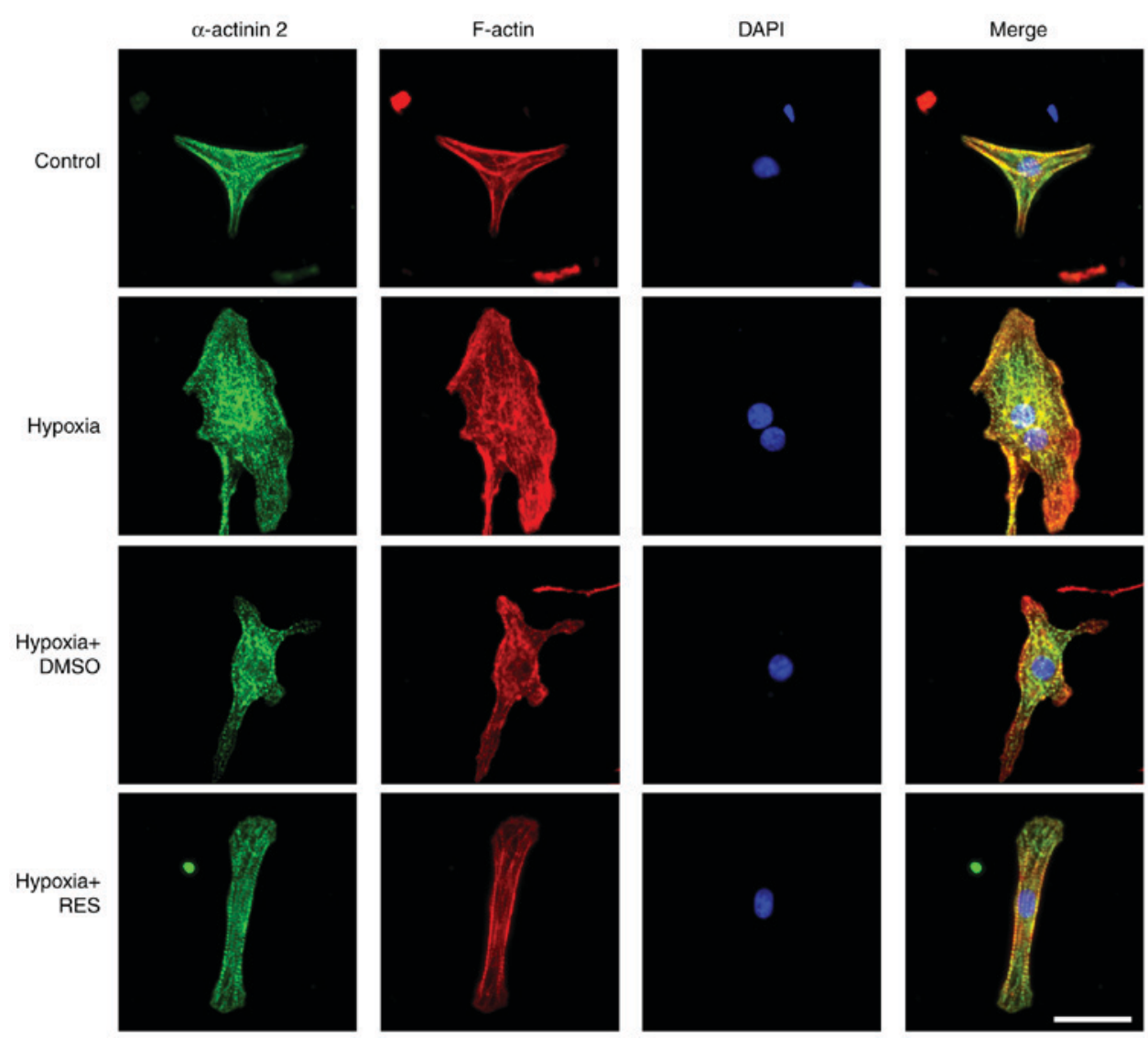

Figure 1. Images of NRCMs with immunofluorescence microscopy showing the attenuating effects of RES on H/R injury-induced structural impairment of NRCMs. $\alpha$-actin (green) and F-actin (red) indicate the T-tubule marker and cytoskeleton marker, respectively. Nuclei were tagged with DAPI (blue). Scale bar=20 $\mu \mathrm{m}$. NRCMs, neonatal rat cardiomyocytes; RES, resveratrol; H/R, hypoxia/reoxygenation; DMSO, dimethyl sulfoxide.

microscope (IX-81, Olympus Corporation, Tokyo, Japan). The excitation wavelengths for DAPI, DyLight ${ }^{\circledR} 488$-conjugated and rhodamine phalloidin were 405,488 and $550 \mathrm{~nm}$, respectively.

Western blotting. The cells were washed with PBS and lysed with lysis buffer containing a cocktail of protease inhibitors (cat. no. 87785, Thermo Fisher Scientific, Inc.). Subsequently, $60 \mu \mathrm{g}$ total protein for each lane was separated with a 5\% stacking gel and $10 \%$ separation gel, and then transferred onto a PVDF membrane (cat. no. IPVH00010; EMD Millipore, Billerica, MA, USA). The membrane was incubated in TBST containing $5 \%$ non-fat milk for $2 \mathrm{~h}$ at room temperature to block non-specific binding and was then incubated with primary antibodies $(1: 1,000)$ overnight at $4^{\circ} \mathrm{C}$. The primary antibodies against Bax (cat. no. 2772; Cell Signaling Technology, Inc., Danvers, MA, USA), Bcl-2 (cat. no. ab196495; Absin, Shanghai, China), Sirt1 (cat. no. 9475; Cell Signaling Technology, Inc.) and the internal control antibody GAPDH (cat. no. sc-25778; Santa Cruz Biotechnology, Inc., Dallas, TX, USA) were polyclonal antibodies raised in rabbit. The membrane was then incubated with horseradish peroxidase (HRP)-conjugated goat anti-rabbit IgG secondary antibody (cat. no. D110058; BBI Life Sciences, Shanghai, China; 1:1,000) for $1 \mathrm{~h}$ at room temperature. The membrane was incubated in chemiluminescent HRP substrate (cat. no. WBKLS0500; EMD Millipore) at room temperature for $5 \mathrm{~min}$, following which images were captured with the Universal Hood II system (Bio-Rad Laboratories, Inc., Hercules, CA, USA).
Measurement of caspase 3 activity. Apoptotic cell death was determined by caspase 3 activation using a Caspase-3 activity assay kit (cat. no. G015, Nanjing Jiancheng Bioengineering Institute). Briefly, the cells were harvested using caspase lysis buffer (50 mM HEPES, pH 7.4, 0.1\% Chaps, $5 \mathrm{mM}$ dithiothreitol, $0.1 \mathrm{mM}$ EDTA and $0.1 \%$ Triton X-100) for $5 \mathrm{~min}$ on ice and centrifuged at $13,000 \mathrm{xg}$ for $10 \mathrm{~min}$ at $4^{\circ} \mathrm{C}$. The superna$\operatorname{tant}(50 \mu \mathrm{g})$ was then isolated and incubated with $10 \mu \mathrm{l}$ caspase 3 substrate (Ac-DEVDpNA) for $1 \mathrm{~h}$ at $37^{\circ} \mathrm{C}$. The activity of caspase 3 was detected with a microplate reader (Tecan Group, Ltd.) at $400 \mathrm{~nm}$.

Statistical analysis. Data are presented as the mean \pm standard error of the mean and were analyzed by one way analysis of variance using SPSS 19.0 (IBM Corp., Armonk, NY, USA). The least significant difference test was used for further multiple group comparisons. $\mathrm{P}<0.05$ was considered to indicate a statistically significant difference.

\section{Results}

RES protects against H/R injury-induced structural impairment in NRCMs. Firstly, the present study investigated the effects of RES on H/R-induced structural impairment in NRCMs using immunohistological staining techniques. As shown in Fig. 1, NRCMs without H/R injury (control group) had a clear morphology with a striated pattern (green) and normal cytoskeletal structure (red). The NRCMs with H/R 

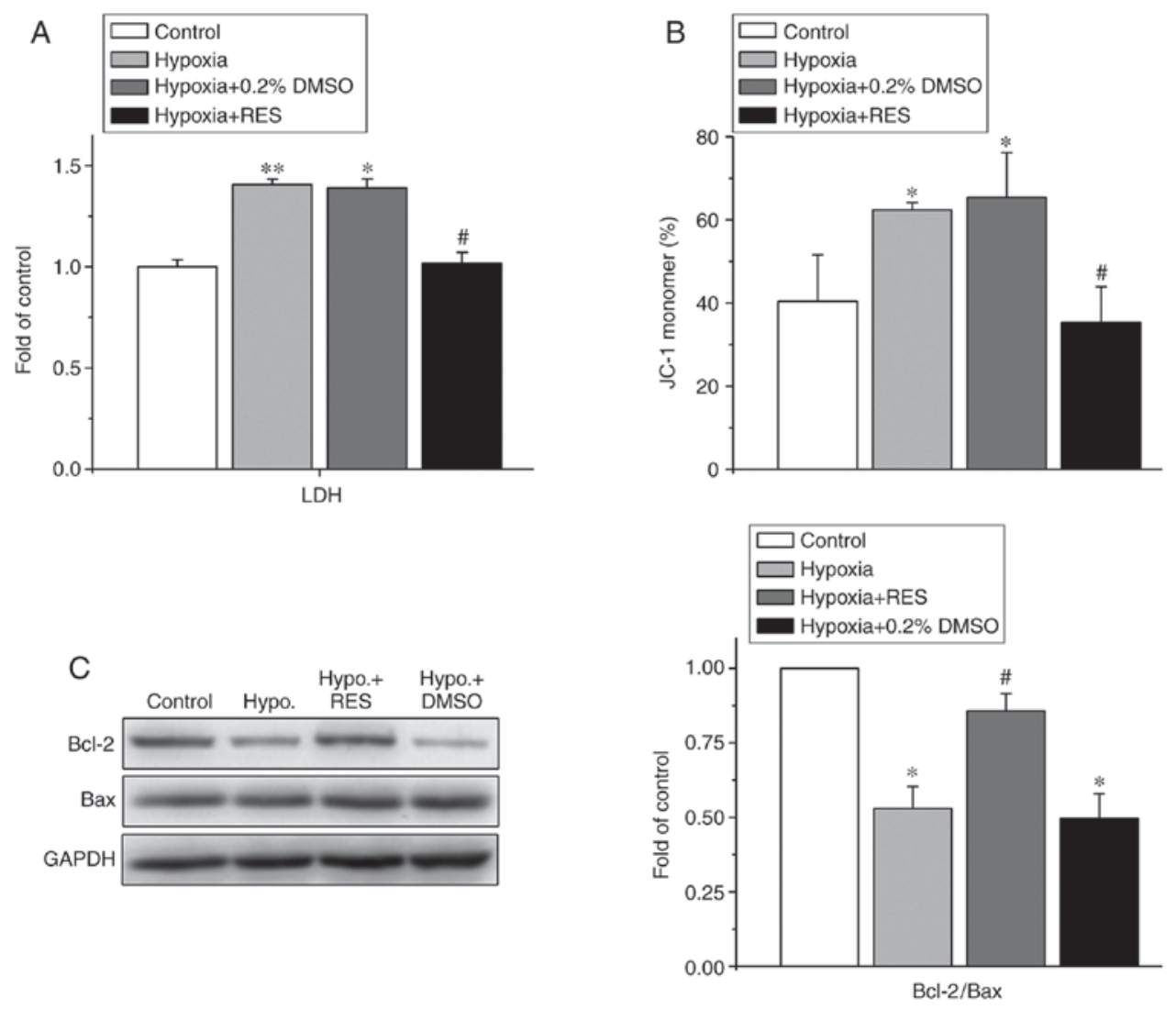

Figure 2. Effects of RES on H/R injury-induced mitochondria oxidative stress in neonatal rat cardiomyocytes. (A) Effect of RES on LDH release ( $n=4$ ). (B) Effect of RES on the $\Delta \Psi \mathrm{m}$, as measured with the JC-1 kit. The percentage of monomer JC-1 showed the level of $\Delta \Psi \mathrm{m}$. The increment of monomer JC-1 represents the depolarization of $\Delta \Psi \mathrm{m}$, whereas the decrement of monomer JC-1 indicates the hyperpolarization of $\Delta \Psi \mathrm{m}$. (C) Western blotting of the protein levels of Bcl-2 and Bax (left) and the ratio of Bcl-2/Bax (right) ( $\mathrm{n}=5$ ). ${ }^{*} \mathrm{P}<0.05$, vs. control; ${ }^{\#} \mathrm{P}<0.05$, vs. H/R treatment. RES, resveratrol; H/R, hypoxia/reoxygenation; LDH, lactate dehydrogenase; $\Delta \Psi \mathrm{m}$, mitochondrial membrane potential; Bcl-2, B-cell lymphoma 2; Bax, Bcl-2-associated X protein; Hypo, hypoxia; DMSO, dimethyl sulfoxide.

injury exhibited significant alterations in morphology and cytoskeletal structure, characterized by disordered $\alpha$-actin and F-actin. In contrast to the NRCMs in the H/R injury group, the impairment of cell structure induced by $H / R$ injury in the NRCMs was significantly attenuated by treatment with $100 \mu \mathrm{M}$ RES. Treatment with $0.2 \%$ DMSO, the final concentration used for diluting RES, did not affect H/R injury-induced structural impairment.

Effects of RES on H/R injury-induced oxidative stress of mitochondria. Mitochondrial oxidative stress is a hallmark of I/R injury in cardiomyocytes. The present study further examined the effect of RES on mitochondrial oxidative stress injury and cell apoptosis, including the determination of LDH release, $\Delta \Psi \mathrm{m}$ and the $\mathrm{Bcl} 2 / \mathrm{Bax}$ ratio. The $\mathrm{Bcl} 2 / \mathrm{Bax}$ ratio in each group was normalized to the value in the control group. As shown in Fig. 2A, exposure to H/R increased LDH release by $1.41 \pm 0.03$-fold compared with that of the control group $(\mathrm{P}<0.01, \mathrm{n}=4)$, whereas $100 \mu \mathrm{M}$ RES significantly attenuated the increased release of LDH induced by $\mathrm{H} / \mathrm{R}$ injury in the NRCMs from $1.41 \pm 0.03$-fold (H/R) to $1.02 \pm 0.06$-fold ( $\mathrm{P}<0.01$, $\mathrm{n}=4)$. Treatment with $0.2 \%$ DMSO did not affect $\mathrm{LDH}$ release when compared with the H/R group. Treatment with H/R induced the depolarization of $\Delta \Psi \mathrm{m}$ by shifting the ratio of JC-1 monomers from $40.43 \pm 11.21 \%$ (control) to $62.39 \pm 1.82 \%$ (H/R) $(\mathrm{P}<0.05, \mathrm{n}=4)$, whereas treatment with $100 \mu \mathrm{M}$ RES alleviated the $\mathrm{H} / \mathrm{R}$-induced depolarization of $\Delta \Psi \mathrm{m}$ by shifting the ratio of JC-1 monomers from $62.39 \pm 1.82 \%(\mathrm{H} / \mathrm{R})$ to $35.31 \pm 8.63 \%$ $(\mathrm{H} / \mathrm{R}+100 \mu \mathrm{M}$ RES $)(\mathrm{P}<0.05, \mathrm{n}=4)$, as shown in Fig. 2B.

Furthermore, the ratio of Bcl2/Bax expression was examined, as detected by western blotting (Fig. 2C). Exposure to $\mathrm{H} / \mathrm{R}$ decreased the $\mathrm{Bcl} 2 / \mathrm{Bax}$ ratio to $0.53 \pm 0.08$-fold of the control $(\mathrm{P}<0.05, \mathrm{n}=5)$, whereas treatment with $100 \mu \mathrm{M}$ RES rescued the $\mathrm{H} / \mathrm{R}$-induced decrease in the $\mathrm{Bcl} 2 / \mathrm{Bax}$ ratio to $0.86 \pm 0.06$-fold $(\mathrm{P}<0.05, \mathrm{n}=5)$.

Effects of RES on H/R-injury-induced apoptosis of NRCMS. The present study also detected the effect of RES on apoptosis induced by $\mathrm{H} / \mathrm{R}$ injury in NRCMs, as shown in Fig. 3. The results of the flow cytometry (Fig. 3A and B) showed that RES attenuated the H/R injury-induced cell apoptosis of NRCMs, with a decrease in the cell apoptotic rate from $84.25 \pm 7.41 \%$ $(\mathrm{H} / \mathrm{R})$ to $46.39 \pm 5.43 \%(\mathrm{H} / \mathrm{R}+\mathrm{RES})(\mathrm{P}<0.05, \mathrm{n}=4)$. Treatment with $0.2 \%$ DMSO alone did not significantly alter the effect of $\mathrm{H} / \mathrm{R}$ on the apoptotic rate of cells $(\mathrm{P}>0.05, \mathrm{n}=4)$.

In addition, caspase 3 is a common downstream signaling molecule involved in cell apoptosis induced by different factors. The present study found that RES alleviated the H/R injury-induced apoptosis of NRCMs (Fig. 3C). The activity of caspase 3 in each group was normalized to the value in the control group. Exposure to H/R increased the activity of caspase 3 to $1.32 \pm 0.06$-fold compared with the control group $(\mathrm{P}<0.05$, 
A
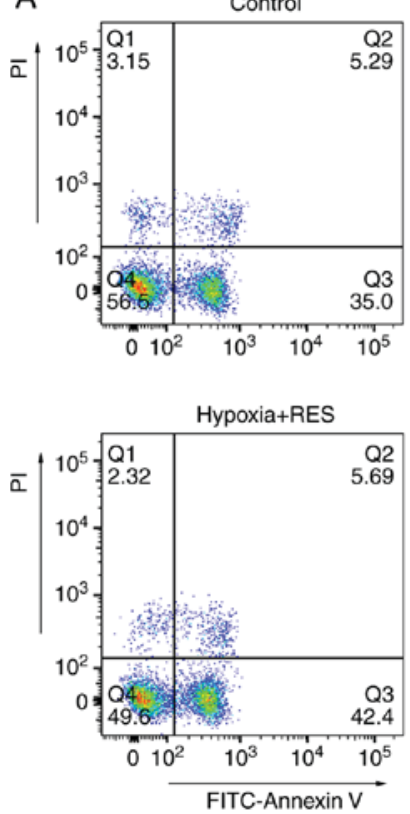
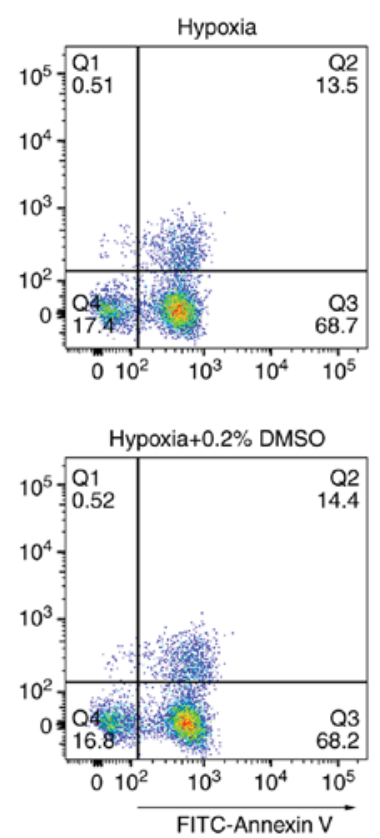
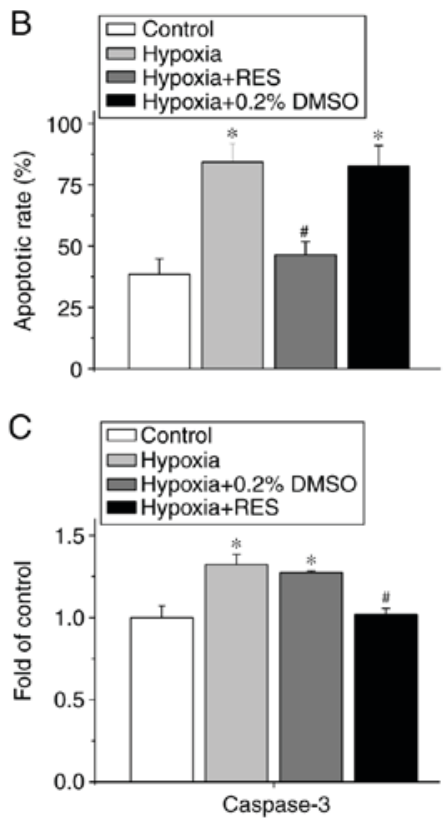

Figure 3. Effects of RES on H/R injury-induced apoptosis of NRCMs. (A) Typical original FCM plots showing the effect of RES on H/R injury-induced apoptosis of NRCMs. FITC Annexin V-and PI-negative cells were viable and did not undergo apoptosis; FITC Annexin V-positive and PI-negative cells were in early stage apoptosis; FITC Annexin V- and PI-positive cells were in the late stage of apoptosis. (B) Statistical bar graph showing the effect of RES on the apoptotic ratio $(\mathrm{n}=4)$. (C) Statistical bar graph showing the activity of caspase 3 in NRCMs that underwent different treatments $(\mathrm{n}=5)$. ${ }^{*} \mathrm{P}<0.05$, vs. control; ${ }^{\#} \mathrm{P}<0.05$, vs. H/R treatment. NRCMs, neonatal rat cardiomyocytes; H/R, hypoxia/reoxygenation; RES, resveratrol; DMSO, dimethyl sulfoxide.

$\mathrm{n}=5)$, whereas treatment with $100 \mu \mathrm{M}$ RES alleviated the increased activity of caspase 3 to $1.02 \pm 0.04$-fold $(\mathrm{P}<0.05, \mathrm{n}=5)$.

Sirtl is involved in the effect of RES on H/R injury in NRCMs. Sirt1 is reportedly an important target of RES, involved in the cardioprotective effects of several drugs indicated for cardiovascular diseases. Therefore, the present study examined whether Sirt1 was involved in the effects of RES on H/R injury in NRCMs. The data are shown in Fig. 4. The expression of Sirt1 in each group was normalized to the value in the control group. Treatment of $\mathrm{H} / \mathrm{R}$ decreased the expression of Sirt1 to $0.61 \pm 0.06$-fold compared with that in the control group $(\mathrm{P}<0.05, \mathrm{n}=5)$, whereas treatment with RES alleviated the decrease of Sirt1 induced by H/R injury to $1.01 \pm 0.05$-fold $(\mathrm{P}<0.05, \mathrm{n}=5)$.

\section{Discussion}

CVDs are the major causes of morbidity and mortality in most countries. I/R injury is a leading cause of myocardial cell death following myocardial infarction. Oxidative stress is the main pathophysiological process involved in I/R injury and other CVDs, including heart failure and hypertension $(23,24)$. Active cardiomyocytes consume energy from the aerobic metabolic pathway, and cardiomyocytes are more sensitive to hypoxia than other cell types. Cardiomyocytes are more prone to metabolic dysfunction, as they are decompensated during the acute I/R condition. Although several studies have verified the cardioprotective effects of RES through inhibiting inflammation and platelet aggregation, alleviating endothelial damage and modulating autophagy, current knowledge is limited regarding the role of oxidative stress injury on RES cardioprotection $(9,25)$. In the present study, an in vitro $\mathrm{H} / \mathrm{R}$
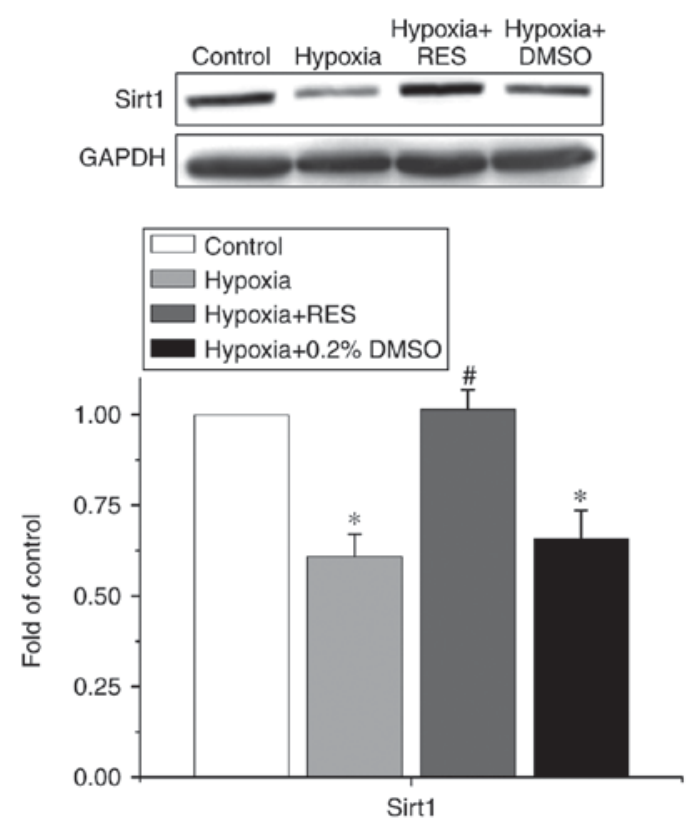

Figure 4. Sirtl is involved in the effect of RES on H/R injury in NRCMs. Results showing the effects on Sirt1. ${ }^{*} \mathrm{P}<0.05$, vs. control; ${ }^{\#} \mathrm{P}<0.05$, vs. $\mathrm{H} / \mathrm{R}$ treatment $(n=5)$. H/R, hypoxia/reoxygenation; RES, resveratrol; Sirt1, sirtuin 1; DMSO, dimethyl sulfoxide.

model was established using NRCMs to imitate I/R injury, and the role of RES on H/R-induced NRCM injury and underlying mechanism were examined. It was found that RES alleviated $\mathrm{H} / \mathrm{R}$-induced NRCM injury and apoptosis through attenuating the mitochondria-mediated oxidative stress pathway.

Dong et al (26) reported that resveratrol protected against pressure-overload-induced cardiac structure injury, and 
exerted beneficial effects on cardiac hypertrophy in a rat model. In the present study, it was found that treatment with RES ameliorated H/R-induced cardiomyocyte structural impairment with F-actin and $\alpha$-actinin 2 , indicating the cytoskeleton and T-tubules (Fig. 1).

Mitochondria are the main organelle involved in biological oxidative reactions. Mitochondria are abundant in cardiomyocytes and the mitochondria-mediated oxidative stress pathway is involved in the cardiac I/R injury process $(5,17)$. $\mathrm{LDH}$ levels, $\Delta \Psi \mathrm{m}$ and the ratio of Bcl-2 to Bax are important indices for reflecting the mitochondria-mediated oxidative stress status (27). The present study found that H/R treatment induced mitochondria oxidative stress, whereas treatment with RES alleviated H/R-induced mitochondrial injury through decreasing the release or $\mathrm{LDH}$, inhibiting the depolarization of $\Delta \Psi \mathrm{m}$ and increasing the ratio of Bcl-2 to Bax (Fig. 2). These data suggest that RES attenuates H/R-induced cardiomyocyte injury through alleviating mitochondria-mediated oxidative stress; mitochondria are targets of RES involved in the cardioprotective effect to attenuate the H/R-induced injury of NRCMs.

The mitochondria-mediated route is an important apoptotic pathway in cells. Enhanced oxidative stress induces cell injury through mitochondria-mediated cell apoptosis. In the present study, it was found that treatment with RES inhibited the apoptosis induced by $\mathrm{H} / \mathrm{R}$ injury through alleviating the cell apoptotic rate and activity of caspase 3, which reflects the status of cell apoptosis (Fig. 3). Therefore, these results support the hypothesis that RES alleviates H/R injury and exerts cardioprotective effects through the mitochondria-mediated signaling pathway.

In addition, Sirt1, a member of the conserved sirtuin family, is an $\mathrm{NAD}^{+}$-dependent histone deacetylase, which is involved in the various cardiac pathophysiological process and cardioprotective effects of certain drugs. Previous studies have suggested that Sirt1 is required for the RES-mediated cardioprotective effect $(28,29)$. Sin et al $(30)$ reported that Sirt1 was involved in the effect of RES for alleviating doxorubicin-induced cardiotoxicity in aged hearts. Recently, Li et al (21) reported that Sirt1 is involved in hypoxia-induced pulmonary artery smooth muscle cell apoptosis. The data obtained in the present study are consistent with those of former studies, and also provide novel evidence that Sirt1 is involved in the cardioprotective effect of RES with respect to $\mathrm{H} / \mathrm{R}$ injury.

The present study found that the cardioprotective effect of RES in H/R-induced cardiomyocyte injury occurs through alleviating mitochondrial oxidative stress and restoring the expression of Sirt1. The results further elucidated the underlying mechanism of RES cardioprotection, and support the widespread clinical use of RES for cardiovascular disease.

\section{Acknowledgements}

Not applicable.

\section{Funding}

This study was supported by the National Natural Science Foundation of China (grant nos. 31300948 and 81670310) and the Joint Foundation of Southwest Medical University and Luzhou city (grant nos. 2015-LZCYD-S03-1/7 and 2016-LZXNYD-T10).

\section{Availability of data and materials}

The datasets and materials used and/or analyzed during the current study are available from the corresponding author on reasonable request.

\section{Authors' contributions}

XQT, TL and LC designed the experiments. TL, LC, YY, BY and PL performed the experiments and collected the data. XQT, TL and LC analyzed and interpreted the data. XQT and TL wrote the manuscript. All authors read and approved the final manuscript.

\section{Ethics approval and consent to participate}

The experimental protocols were performed in accordance with the National Institutes of Health Guide for the Care and Use of Laboratory Animals (National Institutes of Health, Bethesda, MD, USA) and with the approval of the Animal Care and Use Committee of the Southwest Medical University, (Sichuan, China).

\section{Patient consent for publication}

Not applicable.

\section{Competing interests}

The authors declare that they have no competing interests.

\section{References}

1. Murphy E and Steenbergen C: Mechanisms underlying acute protection from cardiac ischemia-reperfusion injury. Physiol Rev 88: 581-609, 2008.

2. Hausenloy DJ and Yellon DM: Myocardial ischemia-reperfusion injury: A neglected therapeutic target. J Clin Invest 123: 92-100, 2013.

3. Paradies G, Paradies V, Ruggiero FM and Petrosillo G: Mitochondrial bioenergetics and cardiolipin alterations in myocardial ischemia/reperfusion injury. Implications for pharmacological cardioprotection. Am J Physiol Heart Circ Physiol: Aug 10, 2018 (Epub ahead of print).

4. Cadenas S: ROS and redox signaling in myocardial ischemia-reperfusion injury and cardioprotection. Free Radic Biol Med 117: 76-89, 2018.

5. Suen DF, Norris KL and Youle RJ: Mitochondrial dynamics and apoptosis. Genes Dev 22: 1577-1590, 2008.

6. von Harsdorf R, Li PF and Dietz R: Signaling pathways in reactive oxygen species-induced cardiomyocyte apoptosis. Circulation 99: 2934-2941, 1999.

7. Bonnefont-Rousselot D: Resveratrol and cardiovascular diseases. Nutrients 8: pii: E250, 2016.

8. Nicholson SK, Tucker GA and Brameld JM: Effects of dietary polyphenols on gene expression in human vascular endothelial cells. Proc Nutr Soc 67: 42-47, 2008

9. Petrovski G, Gurusamy N and Das DK: Resveratrol in cardiovascular health and disease. Ann N Y Acad Sci 1215: 22-33, 2011.

10. Dolinsky VW and Dyck JR: Calorie restriction and resveratrol in cardiovascular health and disease. Biochim Biophys Acta 1812: 1477-1489, 2011. 
11. Ge L, Li C, Wang Z, Zhang Y and Chen L: Suppression of oxidative stress and apoptosis in electrically stimulated neonatal rat cardiomyocytes by resveratrol and underlying mechanisms. J Cardiovasc Pharmacol 70: 396-404, 2017.

12. Meng Z, Jing H, Gan L, Li H and Luo B: Resveratrol attenuated estrogen-deficient-induced cardiac dysfunction: Role of AMPK, SIRT1, and mitochondrial function. Am J Transl Res 8: 2641-2649, 2016

13. Wu Z, Huang A, Yan J, Liu B, Liu Q, Zhang J, Zhang X, Ou C and Chen M: Resveratrol ameliorates cardiac dysfunction by inhibiting apoptosis via the PI3K/Akt/FoxO3a pathway in a rat model of diabetic cardiomyopathy. J Cardiovasc Pharmacol 70: 184-193, 2017.

14. Della-Morte D, Dave KR, DeFazio RA, Bao YC, Raval AP and Perez-Pinzon MA: Resveratrol pretreatment protects rat brain from cerebral ischemic damage via a sirtuin 1-uncoupling protein 2 pathway. Neuroscience 159: 993-1002, 2009.

15. Gurusamy N, Lekli I, Mukherjee S, Ray D, Ahsan MK, Gherghiceanu M, Popescu LM and Das DK: Cardioprotection by resveratrol: A novel mechanism via autophagy involving the mTORC2 pathway. Cardiovasc Res 86: 103-112, 2010.

16. Ma XQ, Fu RF, Feng GQ, Wang ZJ, Ma SG and Weng SA Hypoxia-reoxygenation-induced apoptosis in cultured neonatal rat cardiomyocyets and the protective effect of prostaglandin $\mathrm{E}$. Clin Exp Pharmacol Physiol 32: 1124-1130, 2005.

17. Addabbo F, Montagnani M and Goligorsky MS: Mitochondria and reactive oxygen species. Hypertension 53: 885-892, 2009.

18. Liu XR, Li T, Cao L, Yu YY, Chen LL, Fan XH, Yang BB and Tan XQ: Dexmedetomidine attenuates H2O2-induced neonatal rat cardiomyocytes apoptosis through mitochondria- and ER-medicated oxidative stress pathways. Mol Med Rep 17: 7258-7264, 2018

19. Tatlidede E, Sehirli O, Velioğlu-Oğünc A, Cetinel S, Yeğen BC, Yarat A, Süleymanoğlu S and Sener G: Resveratrol treatment protects against doxorubicin-induced cardiotoxicity by alleviating oxidative damage. Free Radic Res 43: 195-205, 2009.

20. Bargiela D, Burr SP and Chinnery PF: Mitochondria and hypoxia: Metabolic crosstalk in cell-fate decisions. Trends Endocrinol Metab 29: 249-259, 2018.

21. Li F, You Y and Zhu H: 15-HETE protects pulmonary artery smooth muscle cells against apoptosis via SIRT1 regulation during hypoxia. Biomed Pharmacother 108: 325-330, 2018
22. Liu XR, Cao L, Li T, Chen LL, Yu YY, Huang WJ, Liu L and Tan XQ: Propofol attenuates $\mathrm{H}_{2} \mathrm{O}_{2}$-induced oxidative stress and apoptosis via the mitochondria- and ER-medicated pathways in neonatal rat cardiomyocytes. Apoptosis 22: 639-646, 2017.

23. Burgoyne JR, Mongue-Din H, Eaton P and Shah AM: Redox signaling in cardiac physiology and pathology. Circ Res 111: 1091-1106, 2012.

24. Rajendran P, Nandakumar N, Rengarajan T, Palaniswami R, Gnanadhas EN, Lakshminarasaiah U, Gopas J and Nishigaki I: Antioxidants and human diseases. Clin Chim Acta 436: 332-347, 2014.

25. Wang L, Gao M, Chen J, Yang Z, Sun J, Wang Z, Huang X, Yuan T, Shen X and Xian S: Resveratrol ameliorates pressure overload-induced cardiac dysfunction and attenuates autophagy in rats. J Cardiovasc Pharmacol 66: 376-382, 2015.

26. Dong Q, Wu Z, Li X, Yan J, Zhao L, Yang C, Lu J, Deng J and Chen M: Resveratrol ameliorates cardiac dysfunction induced by pressure overload in rats via structural protection and modulation of $\mathrm{Ca}(2+)$ cycling proteins. J Transl Med 12: 323, 2014.

27. Zhou L and Chang DC: Dynamics and structure of the Bax-Bak complex responsible for releasing mitochondrial proteins during apoptosis. J Cell Sci 121: 2186-2196, 2008.

28. Guo R, Liu W, Liu B, Zhang B, Li W and Xu Y: SIRT1 suppresses cardiomyocyte apoptosis in diabetic cardiomyopathy: An insight into endoplasmic reticulum stress response mechanism. Int J Cardiol 191: 36-45, 2015.

29. Chen CJ, Yu W, Fu YC, Wang X, Li JL and Wang W: Resveratrol protects cardiomyocytes from hypoxia-induced apoptosis through the SIRT1-FoxO1 pathway. Biochem Biophys Res Commun 378: 389-393, 2009.

30. Sin TK, Tam BT, Yung BY, Yip SP, Chan LW, Wong CS, Ying M, Rudd JA and Siu PM: Resveratrol protects against doxorubicin-induced cardiotoxicity in aged hearts through the SIRT1-USP7 axis. J Physiol 593: 1887-1899, 2015. 